\title{
Estimation of Discontinuities from Point Cloud Based on Variable-Box Segmentation Method
}

\author{
Shun Matsukawa ${ }^{1}$, Ken-ichi Itakura ${ }^{1}$, Yukinori Suzuki ${ }^{1}$, Akira Hayano ${ }^{2 *}$ \\ ${ }^{1}$ Muroran Institute of Technology, 27-1 Mizumoto-cho, Muroran, Japan \\ ${ }^{2}$ JAEA Horonobe Underground Research Center, Horonobe, Japan; *hayano.akira@jaea.go.jp
}

SNE 28(3), 2018, 121 - 123, DOI: 10.11128/sne.28.sn.10433

Received: Sept. 15, 2016 (Selected EUROSIM Congress 2016

Postconf. Publ.), Revised July 30, Accepted: August 25, 2018

SNE - Simulation Notes Europe, ARGESIM Publisher Vienna,

ISSN Print 2305-9974, Online 2306-0271, www.sne-journal.org

Abstract. For estimating discontinuities of a rock mass from point cloud (LiDAR) data, an algorithm called DiAnahas been used. It obtains a fracture plane from the valid point cloud inside a cubic bounding box. In DiAna, to extract the valid point cloud from the cubic bounding box, DiAna has to determine the threshold value to remove noises. It seems that this manual operation is hard work for long tunnels. To improve this manual operation, we devel-oped Variable-Box Segmentation (VBS) method for LiDAR data recorded from a tunnel. VBS has three processes, i.e., first segmentation, second segmentation, and combining. During the first segmentation, the point cloud is segmented into large bounding boxes and noise is removed. During the second segmentation, each box is divided into nine sub-boxies. Planes are estimated from point cloud data inside each sub-box. During combining, sub-boxies containing similar planes are joined. VBS was examined using LiDAR data including three sets of major discontinuities. Estimation results from VBS was compared with the reference planes decided from geological sketch. Results showed that similarity between reference planes and planes determined by VBS algorithm seems to be enough to find discontinuities from fractured planes..

\section{Introduction}

To construct the tunnel, geological mappings on the rock wall and faces by geologists are important for the design of optimal support, maintenance, and managing the tunnel for a long life cycle.
However, in a long tunnel, construction costs have become higher. Geologists must work under an unstable gallery for a long time. Furthermore, fracture maps by geologists can change individually. To avoid such problems, 3D laser scanning to acquire a high-resolution point cloud (LiDAR) has been introduced for tunnel construction (Fekete et al., 2010; Lato et al., 2009). In fact, LiDAR can provide detailed 3D characteristics and structures of the rock around the tunnel, along with information related to the rock discontinuity. In previous studies, some methods to estimate discontinuity from LiDAR were developed. Diederichs et al. (Lato et al., 2009) reconstructed surfaces using a polygonal model and grouped them as co-planar. Lato et al. (Vöge et al., 2013) developed "PlaneDetect", which consists of five processing stages: smoothing, edge detection, blast damage detection, discontinuity identification, and discontinuity set clustering.

Developed by Gigli et al. (Gigli and Casagli, 2011), DiAna is an algorithm used to estimate the discontinuities in a rock mass from facets semi-automatically. It segments the point cloud into cubic bounding boxes with fixed size and ascertains the plane of points using least squares method. When DiAna extracts the valid point cloud from the cubic bounding box, it has to determine the threshold value based on the standard deviation of distances between the points and plane in the box manually. However, it seems that this manual operation is hard work for long tunnels. To improve this manual operation, we developed Variable-Box Segmentation (VBS) method for LiDAR data recorded from the tunnel.

This paper presents Variable-Box Segmentation (VBS) method to produce suitable planes for estimating discontinuity in the tunnel automatically from a point cloud. VBS segments the point cloud into parallelepiped bounding boxes. Then it combines boxes to produce suitable planes at each part of the point cloud. 


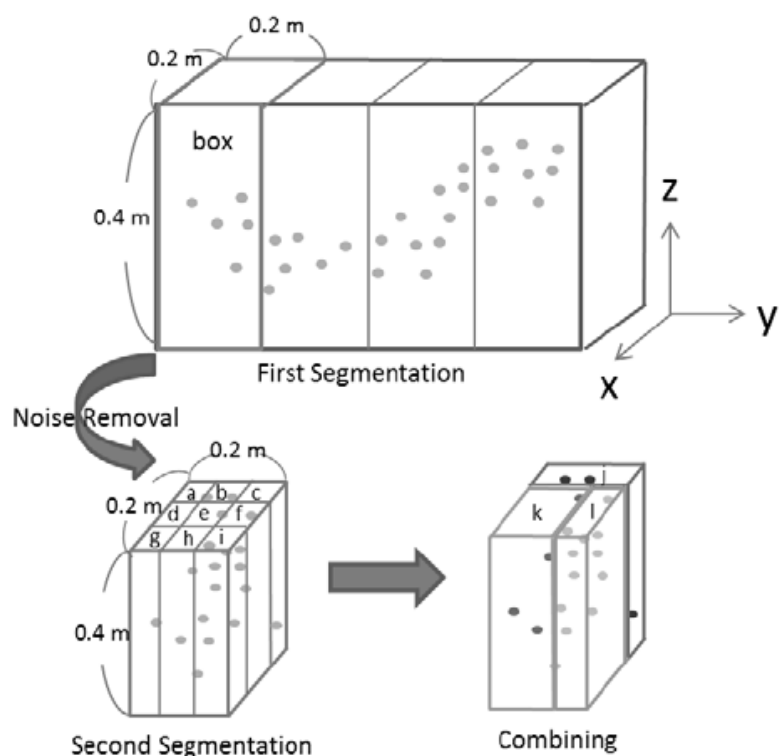

Figure 1. VBS algorithm. First, segment into a large box and remove noise (upper, red box). Next segment into nine sub-boxes a-i (lower left, sub-boxes). Finally, combine sub-boxes to produce suitable planes, e.g. j, $\mathrm{k}$ and I (lower right, purple, blue or green boxes).

In Section 1, details of the VBA algorithm are presented. Experimental results of VBA using the point cloud from LiDAR are presented in Section 2. Finally, this paper is concluded in Section 3.

\section{VBS Algorithm}

VBS has three processes as shown in Figure 1: first segmentation, second segmentation, and combining. In the first segmentation, the point cloud is segmented into large bounding boxes $(0.2 \times 0.2 \times 0.4 \mathrm{~m})$. Noise is removed from the boxes. To remove noise, each box is segmented again into mini-boxes $(0.02 \times 0.02 \times$ $0.02 \mathrm{~m}$ ). The points in that box are probably noise if the mini-boxes are sparse. Then VBS sums up the contained number of points from the sparsest box to the densest box until the summation becomes greater than $31.73 \%$ of all points. Then VBS removes the summed points as noise. This border of the summation is determined empirically. In the second segmentation, noise removed boxes are segmented into nine sub-boxes (smaller boxes) as shown in Figure 1. Planes are obtained from each sub-box using least squares method.
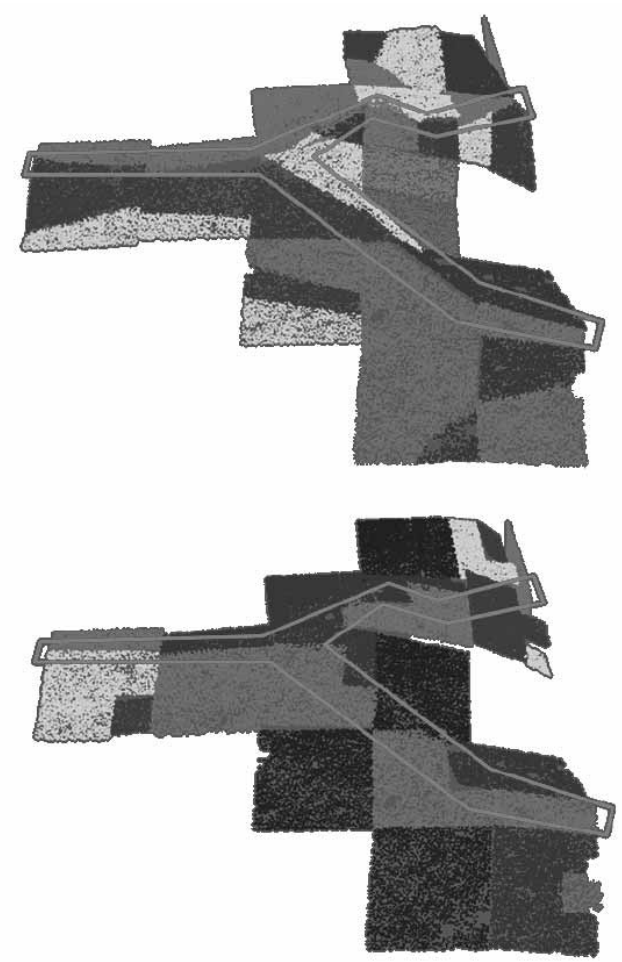

Figure 2. The images show planes for discontinuity 1. Upper and lower panels are the reference planes and those of planes determined by VBS algorithm. Color shows each planes. Red frames show the discontinuity.

Combining the process, each sub-box that has a similar normal vector of plane is combined as shown in Figure 1. The distances between the plane and points are computed for all points in the sub-box. If the box contains more than $68.27 \%$ of all points that is distance inside of the standard deviation, then the plane is combined by the region growing method. If not, the box is combined with the most similar box among its four neighbors.

\section{Examination and Results}

VBS has been examined using gallery data collected from the $-300 \mathrm{~m}$ Access/Research Gallery of the Mizunami Underground Research Laboratory using a LiDAR scanner. The examination was conducted for the following processes: First, three major fracture discontinuities (discontinuity 1 , discontinuity 2 , and discontinuity 3 ) observed by geological engineers were extracted from point cloud datasets and we obtained reference discontinuity planes (remove noises and make a cluster from large boxes, then compute these planes) manually. 
Next, planes were computed from the same datasets using VBS for $0.2 \times 0.2 \times 0.4(\mathrm{~m})$ boxes were calculated. The VBS results were compared with reference planes by geological sketch observations and cosine distances.

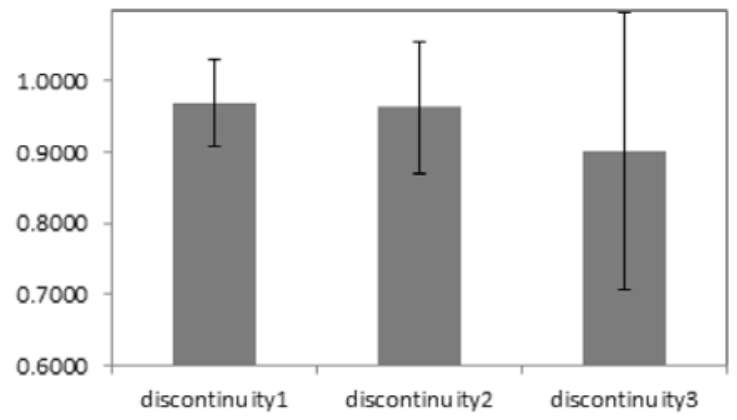

Figure 3. Average cosine distances with its standard deviation between reference planes and planes determined by VBS algorithm for three fracture discontinuities. discontinuity1: 0.9700 \pm 0.0617 , discontinuity2: $0.9631 \pm 0.0929$, discontinuity3: $0.9020 \pm 0.1942$.

Results of the discontinuity are presented in Figure 2 and Figure 3. The image in the upper in Figure 2 presents the reference planes. The image in the lower Figure 2 also presents the planes determined by VBS algorithm. In Sketch observation reveals that lower image describes the boundary of the discontinuity areas well fitting the reference. Figure 3 shows the average cosine distances between the normal vector of reference plane and that of plane determined by the developed algorithm. Since the cosine distances for all fracture discontinuities are more than 0.900 , the planes determined by VBS algorithm are enough similar to reference planes. From observing the geological sketch and cosine distances we can reason that planes determined by the developed algorithm seem to be enough similar to the reference planes to find discontinuities.

\section{Conclusion}

To estimate discontinuities of rocks around a tunnel from a point cloud, an algorithm called DiAna is effective. To extract the valid point cloud from the cubic bounding box, DiAna determined the threshold values manually. It seems to be hard work for long tunnels. To improve this manual operation, we developed VariableBox Segmentation (VBS) method for LiDAR data recorded from the tunnel. Similarity between reference planes and planes determined by the developed algorithm seems to be enough to find discontinuities from fractured planes.

\section{References}

[1] Fekete S, Diederichs M, and Lato M. Geotechnical and operational applications for three-dimensional laser scanning in drill and blast tunnels. Tunnelling and Underground Space Technology, 25:614-628, 2010.

[2] Gigli G and Casagli N. Semi-automatic extraction of rock mass structural data from high resolution lidar point clouds. Inter-national Journal of Rock Mechanics and Mining Sciences, 48 (2):187-198, 2011.

[3] Lato M, Diederichs MS, Hutchinson DJ, and Harrap R. Optimization of lidar scanning and processing for automated structural evaluation of discontinuities in rockmasses. International Journal of Rock Mechanics and Mining Sciences, 46 (1):194-199, 2009.

[4] Vöge M, Lato MJ, and Diederichs MS. Automated rockmass discontinuity mapping from three-dimensional surface data. Engineering Geology, 164:155-162, 2013. 